ARTICLE

\title{
Fermion-boson many-body interplay in a frustrated kagome paramagnet
}

J.-X. Yin (10) 1,15凶, Nana Shumiya ${ }^{1,15}$, Sougata Mardanya (1) 2,15, Qi Wang ${ }^{3,15}$, Songtian S. Zhang (1) 1,15, Hung-Ju Tien ${ }^{2,15}$, Daniel Multer ${ }^{1}$, Yuxiao Jiang${ }^{1}$, Guangming Cheng ${ }^{4}$, Nan Yao ${ }^{4}$, Shangfei $\mathrm{Wu}^{5}$, Desheng $\mathrm{Wu}^{5}$,

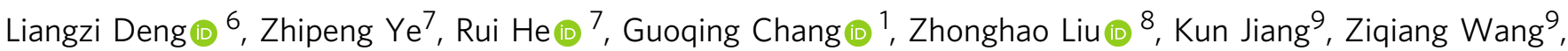
Titus Neupert (10) ${ }^{10}$, Amit Agarwal ${ }^{11}$, Tay-Rong Chang $2,12,13$, Ching-Wu Chu ${ }^{6,14}$, Hechang Lei (i) ${ }^{3} \&$ M. Zahid Hasan (1) 1,14凶

Kagome-nets, appearing in electronic, photonic and cold-atom systems, host frustrated fermionic and bosonic excitations. However, it is rare to find a system to study their fermion-boson many-body interplay. Here we use state-of-the-art scanning tunneling microscopy/spectroscopy to discover unusual electronic coupling to flat-band phonons in a layered kagome paramagnet, CoSn. We image the kagome structure with unprecedented atomic resolution and observe the striking bosonic mode interacting with dispersive kagome electrons near the Fermi surface. At this mode energy, the fermionic quasi-particle dispersion exhibits a pronounced renormalization, signaling a giant coupling to bosons. Through the selfenergy analysis, first-principles calculation, and a lattice vibration model, we present evidence that this mode arises from the geometrically frustrated phonon flat-band, which is the lattice bosonic analog of the kagome electron flat-band. Our findings provide the first example of kagome bosonic mode (flat-band phonon) in electronic excitations and its strong interaction with fermionic degrees of freedom in kagome-net materials.

\footnotetext{
${ }^{1}$ Laboratory for Topological Quantum Matter and Spectroscopy (B7), Department of Physics, Princeton University, Princeton, NJ 08544, USA. ${ }^{2}$ Department of Physics, National Cheng Kung University, 701 Tainan, Taiwan. ${ }^{3}$ Department of Physics and Beijing Key Laboratory of Opto-electronic Functional Materials\&Micro-nano Devices, Renmin University of China, 100872 Beijing, China. ${ }^{4}$ Princeton Institute for Science and Technology of Materials (PRISM), Princeton University, Princeton, NJ 08544, USA. ${ }^{5}$ Institute of Physics, Chinese Academy of Sciences, 100190 Beijing, China. ${ }^{6}$ Department of Physics and Texas Center for Superconductivity, University of Houston, Houston, TX 77204-5002, USA. 7 Department of Electrical and Computer Engineering, Texas Tech University, Lubbock, TX 79409, USA. ${ }^{8}$ State Key Laboratory of Functional Materials for Informatics and Center for Excellence in Superconducting Electronics, Shanghai Institute of Microsystem and Information Technology, Chinese Academy of Sciences, 200050 Shanghai, China. ${ }^{9}$ Department of Physics, Boston College, Chestnut Hill, MA 02467, USA. ${ }^{10}$ Department of Physics, University of Zurich, Winterthurerstrasse 190, Zurich, Switzerland. ${ }^{11}$ Department of Physics, Indian Institute of Technology Kanpur, Kanpur 208016, India. ${ }^{12}$ Center for Quantum Frontiers of Research and Technology (QFort), 701 Tainan, Taiwan. ${ }^{13}$ Physics Division, National Center for Theoretical Sciences, 30013 Hsinchu, Taiwan. ${ }^{14}$ Material Sciences Division, Lawrence Berkeley National Laboratory, Berkeley, CA 94720, USA. ${ }^{15}$ These authors contributed equally: J.-X. Yin, Nana Shumiya, Sougata Mardanya, Qi Wang, Songtian S.

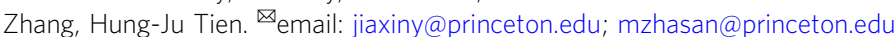


T he kagome-net, a pattern of corner-sharing triangular plaquettes, has been a fundamental model platform for exotic states of matter, including quantum spin liquids and topological band structures ${ }^{1-3}$. Recently, the transition metal-based kagome metals ${ }^{4-13}$ are emerging as a new class of topological quantum materials to explore the interplay between frustrated lattice geometry, nontrivial band topology, symmetry-breaking order, and many-body interaction. A kagome lattice tight-binding model generically features a Dirac crossing and a flat-band, which are the fundamental sources of nontrivial topology and strong correlation. Such topological fermionic structures arising from the correlated $3 d$ electrons in the kagome lattice have been widely reported in several quantum materials ${ }^{4-13}$, including $\mathrm{Mn}_{3} \mathrm{Sn}, \quad \mathrm{Fe}_{3} \mathrm{Sn}_{2}, \mathrm{Co}_{3} \mathrm{Sn}_{2} \mathrm{~S}_{2}$, $\mathrm{TbMn}_{6} \mathrm{Sn}_{6}, \mathrm{FeSn}$, and CoSn. In parallel, the band dispersion of bosonic excitations on a kagome lattice also features Dirac crossings and flat-bands, as demonstrated in photonic crystals ${ }^{14,15}$. A question naturally arising when studying kagome lattice electrons is the possibility of a nontrivial many-body interplay between the bosonic kagome lattice phonons and the fermionic quasiparticles.

Such fermion-boson interactions often manifest as a perturbation of the bare band structures at very low-energy scales. However, most kagome lattice materials exhibit complicated multi-bands near the Fermi energy ${ }^{4-12}$, severely challenging the clear identification of the many-body effect by spectroscopic methods. Among all known kagome lattice materials, the paramagnetic CoSn is recently highlighted as an outstanding kagome topological metal with much cleaner bands and simpler Fermi surface ${ }^{13}$, making it an ideal platform to search for the geometrical frustrated fermion-boson interaction. Here we report the discovery of fermion-boson many-body interplay in kagome lattice of CoSn, which arises from the coupling of the phonon flat-band with the kagome electrons, utilizing the low-temperature $(T=4.2 \mathrm{~K})$, high energy-resolution $(\Delta E<0.3 \mathrm{meV})$, atomic layer-resolved scanning tunneling microscopy.

\section{Results}

Atomic-scale visualization of kagome lattice. CoSn has a hexagonal structure (space group $\mathrm{P} 6 / \mathrm{mmm}$ ) with lattice constants ${ }^{13,16}$ $a=5.3 \AA$ and $c=4.4 \AA$. It consists of a $\mathrm{Co}_{3} \mathrm{Sn}$ kagome layer and an $\mathrm{Sn}_{2}$ honeycomb layer (Fig. 1a) with alternating stacking. The side-plane atomically resolved map of the crystal measured by transmission electron microscopy (Fig. 1b) directly demonstrates this atomic stacking sequence along the $c$ axis. Upon cryogenic cleaving, the surface yields either the $\mathrm{Co}_{3} \mathrm{Sn}$ - or $\mathrm{Sn}_{2}$-terminated atomic layer. The lower panel in Fig. 1c shows a highly rare topographic image of the cleaving surface that contains both terminations. It consists of a $\mathrm{Co}_{3} \mathrm{Sn}$ surface and islands of $\mathrm{Sn}_{2}$ layer sitting on top. The simultaneously obtained differential conductance map directly reveals their difference in the electronic structure shown in the upper panel of Fig. 1c, where the $\mathrm{Co}_{3} \mathrm{Sn}$ surface has a higher density of states at the bias voltage of $100 \mathrm{mV}$. From this map, we also find that the $\mathrm{Co}_{3} \mathrm{Sn}$ surface has detectable impurities induced quasi-particle interferences, which is the basis for our further space-momentum investigation. Scanning the $\mathrm{Sn}_{2}$ (Fig. 1d) and $\mathrm{Co}_{3} \mathrm{Sn}$ (Fig. 1e) surfaces with higher magnification, we directly reveal their honeycomb and kagome lattice symmetry, respectively. Remarkably, our topographic image was able to resolve the fine corner-sharing triangle structure of the Co kagome lattice and the Sn atom in the kagome center (Fig. 1e). Such ultrahigh atomic resolution has not been achieved in the previous scanning tunneling studies of kagome lattice materials.

Bosonic mode coupling from kagome electrons. With the lattice structure of CoSn visualized at the atomic scale, we now study their electronic structure by measuring the differential conductance as shown in Fig. 2a. According to the first-principle calculations and photoemission measurement, the Fermi surface is dominated by a fairly simple electron-like band ${ }^{13}$. Strikingly, we find pronounced low-energy modulations for the spectra taken on the $\mathrm{Co}_{3} \mathrm{Sn}$ layer, while this feature is absent on the $\mathrm{Sn}_{2}$ layer as shown in Fig. $2 \mathrm{~b}$. The observed peak-dip-hump modulation can indicate the strong bosonic mode coupling with the coherent state at the Fermi level, as similar spectroscopic features have been found in many strong coupling superconductors, including lead ${ }^{17}$, cuprates ${ }^{18}$, and iron-pnictides ${ }^{19}$. Such a bosonic mode often arises from phonons or spin resonances. As this material is nonmagnetic and has no detectable magnetic field dependence of tunneling spectra up to $8 \mathrm{~T}$ (Fig. 2c), the bosonic a

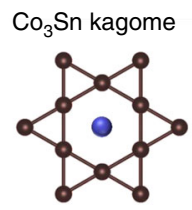

b
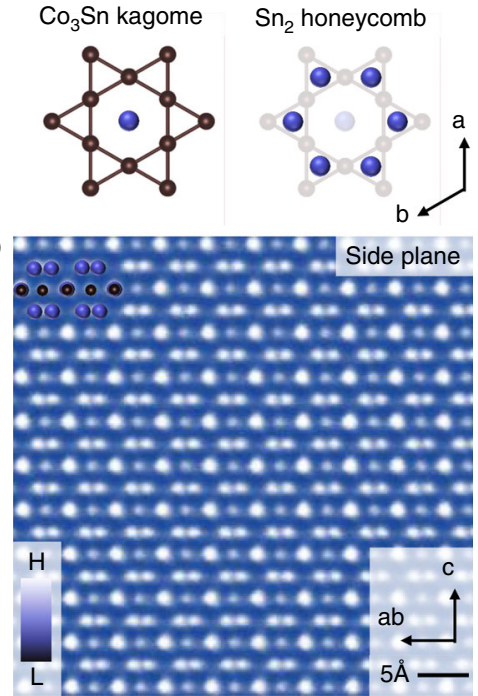

\section{e}

c

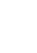

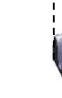

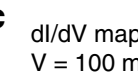

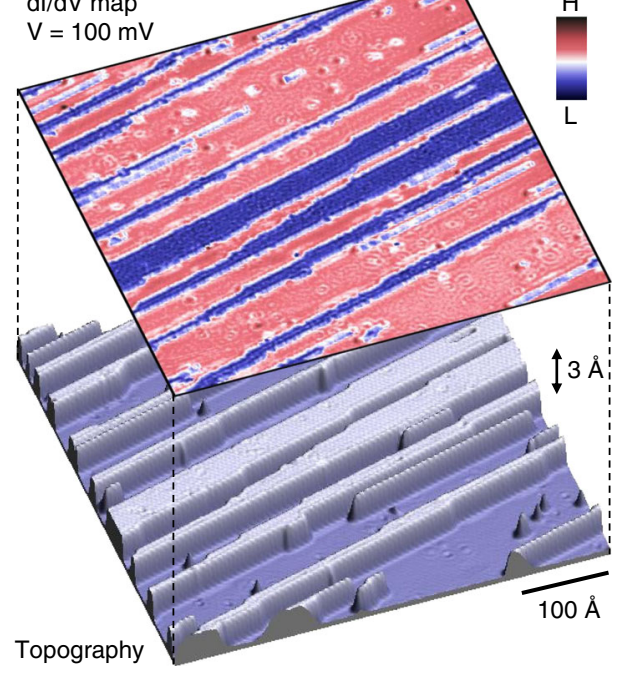

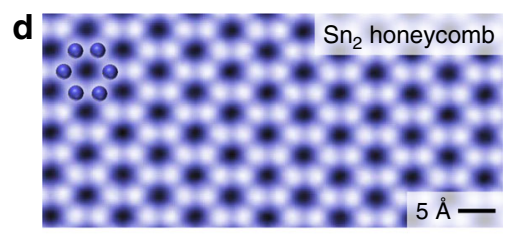

e

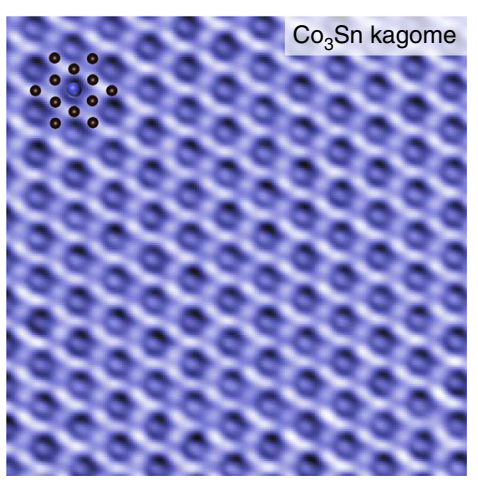

Fig. 1 Complete atomic-scale visualization of kagome lattice in CoSn. a Crystal structure of CoSn, which consists of a $\mathrm{Co}_{3} \mathrm{Sn}_{\mathrm{kagome}}$ layer (left) and a Sn honeycomb layer (right). b Cross-sectional atomic resolution scanning transmission electron microscope image of CoSn, showing the lattice stacking along the $c$ axis as illustrated in the inset. c Topographic image of a single atomic step (bottom) and the corresponding differential conductance map obtained at a bias voltage of $100 \mathrm{mV}$ (top). d Atomically resolved $\mathrm{Sn}_{2}$ surface with the corresponding atomic lattice structures. e Atomically resolved $\mathrm{Co}_{3} \mathrm{Sn}$ surface with the corresponding atomic lattice structures. 

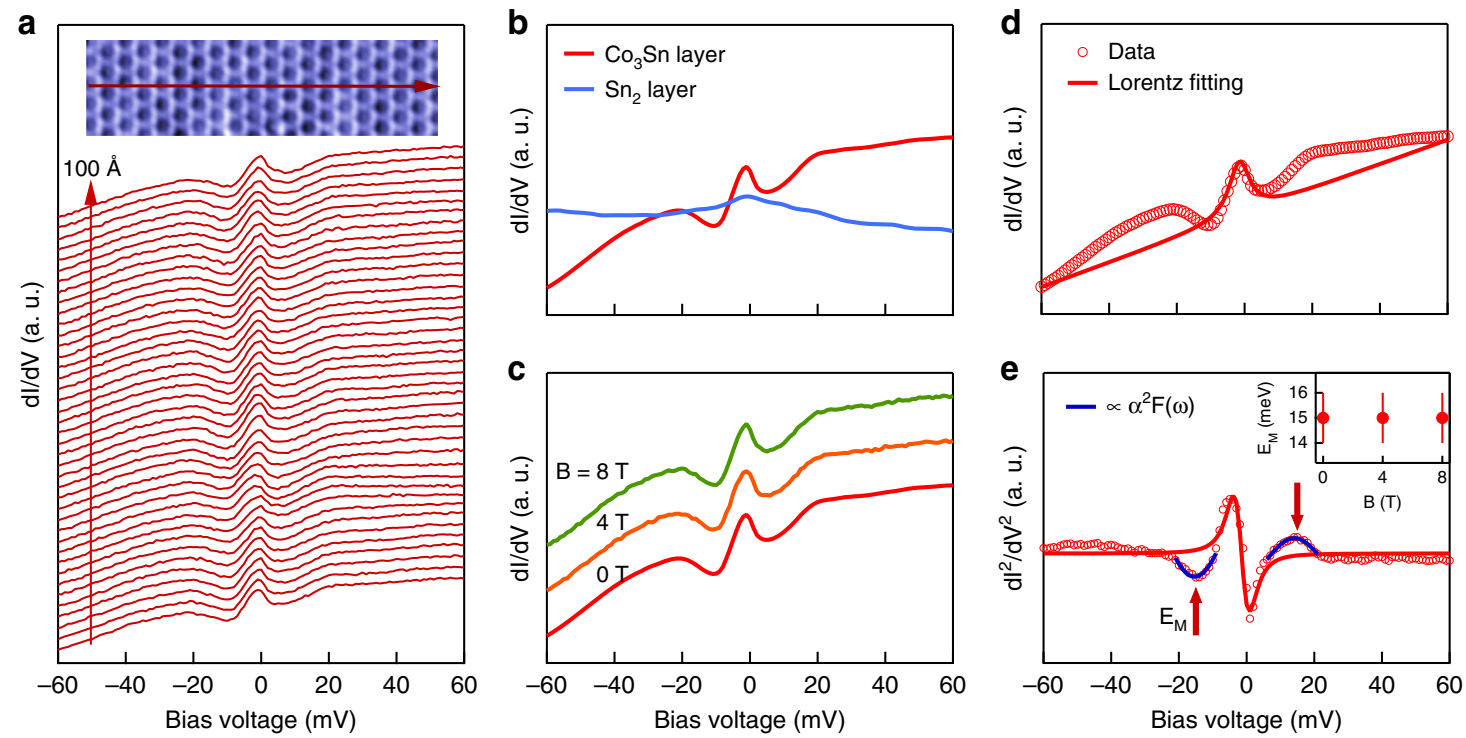

Fig. 2 Bosonic mode coupling from kagome electrons. a Differential conductance spectra taken along the arrow draw on the $\mathrm{CO}_{3} \mathrm{Sn}$ layer shown in the inset. b Spatially averaged $\mathrm{d} l / \mathrm{d} V$ spectra for $\mathrm{Co}_{3} \mathrm{Sn}$ layer and $\mathrm{Sn}_{2}$ layer, respectively. $\mathbf{c} \mathrm{d} / \mathrm{d} V$ spectrum for $\mathrm{Co}_{3} \mathrm{Sn}$ layer taken at different external magnetic fields applied along the $\mathrm{c}$ axis. $\mathbf{d}$ Fitting of the spectral peak in the $\mathrm{Co}_{3} \mathrm{Sn}$ layer with a Lorentzian. $\mathbf{e}$ Second differential conductance for the data in $\mathbf{d}$ with the extra Gaussian-like peaks marked by blue lines centered at the energy $E_{M}= \pm 15$ meV marked by red arrows. The inset plot shows the $E_{M}$ value is independent of the external magnetic field.

mode is more likely to arise from the coupling to phonons. Moreover, the electronic coupling to the bosonic mode can be described within the Eliashberg theory ${ }^{20,21}$ with $\alpha^{2} F(\omega)$ where $\alpha$ is the coupling matrix element and $F(\omega)$ is the bosonic density of states, and this can be studied in the tunneling experiments. $\alpha^{2} F(\omega)$ is intimately related to the second differentiation of the tunneling spectra ${ }^{17-22}$. Analyzing the measured tunneling spectra (Fig. 2d, e), we find a Gaussian-like state in the second derivative centered at $E_{\mathrm{M}}=15 \mathrm{meV}$ with a full width at half maximum of $9 \mathrm{meV}$ (Fig. 2e), and identify it as a candidate signal related to the Eliashberg function (Fig. 2e, blue curves). The other peak features at lower energies of the second differential spectra can be expected from a coherent state at the Fermi energy as shown by the simulation curve in Fig. 2 d, e.

Many-body kink in the kagome dispersion. To gain deeper insight into the bosonic mode coupling on the kagome lattice, we perform systematic spectroscopic imaging on a large $\mathrm{Co}_{3} \mathrm{Sn}$ area with only a few $\mathrm{Sn}_{2}$ adatoms (Fig. 3a). By taking the Fourier transform of the differential conductance map (Fig. 3b), we obtain the quasi-particle interference (QPI) data. The QPI data at $0 \mathrm{meV}$ (Fig. 3c) shows a clear ring-like signal, consistent with the intraband scattering of the dominant electron-like Fermi surface $^{13}$ centered at $\Gamma$. Thus, the low-energy QPI dispersion reflects the behavior of the electron-like band crossing the Fermi surface $(Q=2 k)$. Analyzing the QPI dispersion along $\Gamma-\mathrm{M}$ direction in Fig. 3d, we observe a pronounced double kink feature, different from its bare band dispersion calculated by the first-principles (dashed line). The spectroscopic kink feature has been identified as a fingerprint of the bosonic mode coupling ${ }^{23-29}$ and indicates a giant mode coupling strength. The energy of the QPI kink is around $\pm 15 \mathrm{meV}$, well consistent with the mode energy $E_{\mathrm{M}}$ in the second differential conductance signal. The coupling strength can be estimated from Fermi velocity renormalization $\lambda=v_{\mathrm{f} 0} / v_{\mathrm{f}}-1=1.8$ with s.d. error bar of 0.3 , where $v_{\mathrm{fo}}$ and $v_{\mathrm{f}}$ are the Fermi velocities of the bare QPI band and renormalized QPI band, respectively. We also explored the QPI on the $\mathrm{Sn}_{2}$ honeycomb lattice but did not find any clear kink. Hence the unique kagome lattice resolving capability combined with low temperature and high energy-resolution of our advanced spectroscopic technique can be the key for the kink discovery in this material.

\section{Discussion}

The pronounced kink signal from the kagome lattice allows us to analyze the electron many-body self-energy $\Sigma(\omega)$ and further quantify the Eliashberg function $\alpha^{2} F(\omega)$. $\Sigma(\omega)$ is intimately related to $\alpha^{2} F(\omega)$, the Fermi-Dirac distribution $f(E)$ and the Bose-Einstein distribution $n(\omega)$ (see Eq. (4) in "Methods" section). The real part of the self-energy $\operatorname{Re}(\Sigma)$ is related to the energy difference between the observed kink dispersion and the bare QPI dispersion, while the imaginary part of the self-energy $\operatorname{Im}(\Sigma)$ is related to the electron band broadening, which is inversely proportional to the QPI intensity. $\operatorname{Re}(\Sigma)$ and $\operatorname{Im}(\Sigma)$ are tied to one another through the Kramers-Kronig relation, and we can more accurately acquire $\operatorname{Re}(\Sigma)$ from the QPI data in Fig. 3e. We take the shape of $\alpha^{2} F(\omega)$ in reference to the second differential conductance (Fig. 2), and tune the coupling strength $\lambda=$ $\int \alpha^{2} F(\omega) / \omega \mathrm{d} \omega$ and calculate the real part of the self-energy $\operatorname{Re}$ $(\Sigma)$. We find that with $\lambda=1.9$ with s.d. error bar of 0.3 , the calculated $\operatorname{Re}(\Sigma)$ can account for that determined by the experiment (Fig. 3e), which agrees with the estimated $\lambda$ from Fermi velocity renormalization. We further simulate the QPI signal with this $\alpha^{2} F(\omega)$ under the Green function formalism in Fig. 3f, which also shows reasonable consistency with the experimental data both in dispersion and intensity evolution, providing key support to our many-body analysis of the kagome fermion-boson interaction.

Having characterized the many-body fermion-boson interaction in the kagome lattice, we perform first-principles calculations of the phonon band to understand the nature of the bosonic mode. Firstly, the calculated phonon density of states exhibits a pronounced peak at $15 \mathrm{meV}$ (Fig. 4a), coincide with the mode energy in experiments. Secondly, this phonon mode mainly arises from the $\mathrm{Co}_{3} \mathrm{Sn}$ kagome layer, consistent with the experiments. Thirdly, the calculation provides momentum space insight into the origin of this peak, in that it arises from a flat-band in phonon 
a
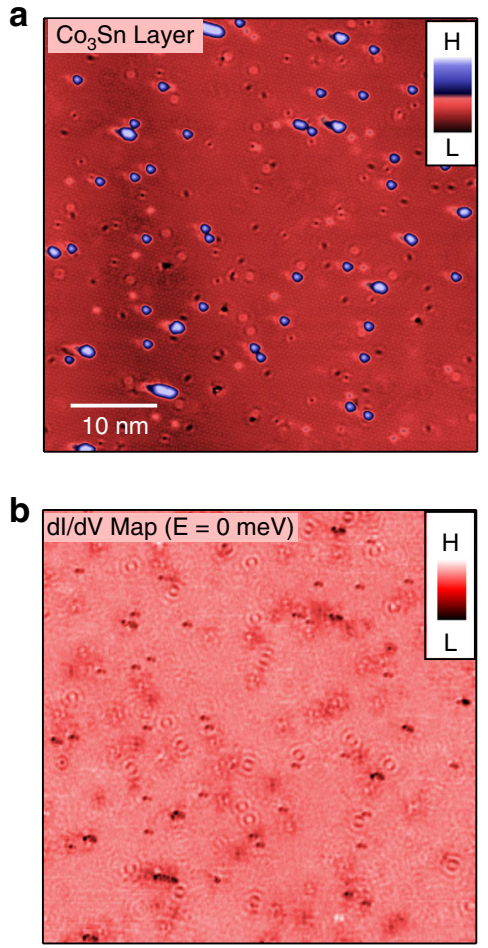

C
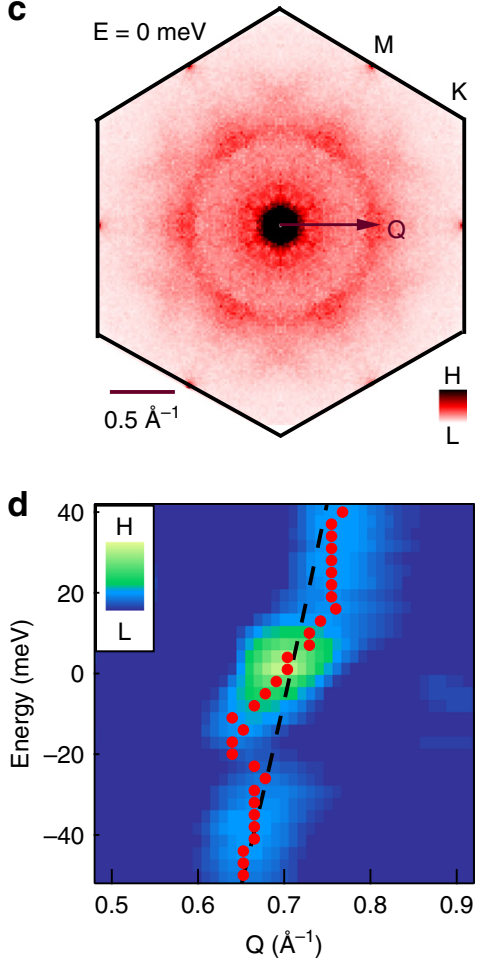
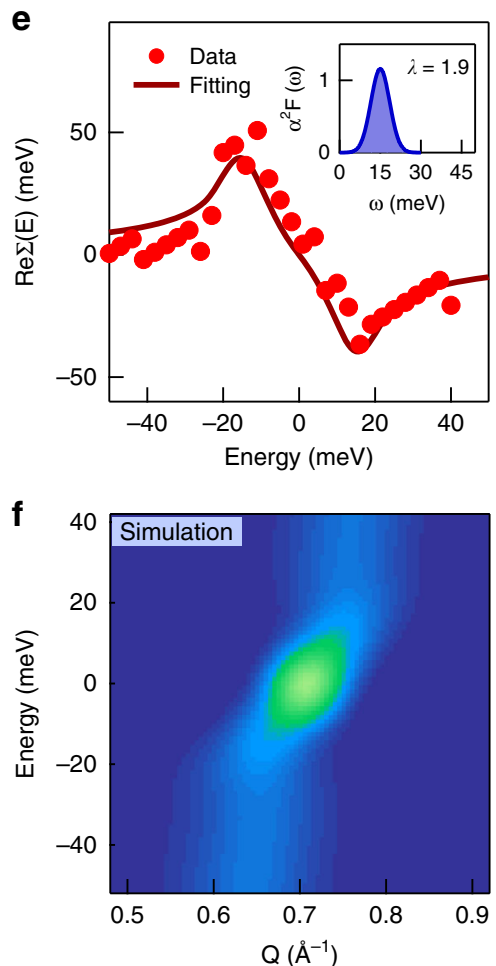

Fig. 3 Bosonic mode induced many-body kink in the kagome dispersion. a A topographic image of $\mathrm{CO}_{3} \mathrm{Sn}_{n}$ layer. b Corresponding differential conductance map taking at $E=0 \mathrm{meV}$. c QPI data of the $\mathrm{Co}_{3} \mathrm{Sn}$ surface. Data have been sixfold symmetrized to enhance the signal to noise ratio. Due to intraband scattering nature, $Q=2 \mathrm{k}$. d QPI dispersion along the $\Gamma-\mathrm{M}$ direction showing a pronounced kink. The dots mark the QPI peak positions while the dashed line illustrates the bare band based on first-principles calculations. e The real part of the electron self-energy. The dots are extracted from the energy difference between the renormalized QPI dispersion and the bare QPI dispersion. The line is calculated based on a Gaussian-like Eliashberg function with coupling strength $\lambda=1.9$ shown in the inset. $\mathbf{f}$ Simulated QPI dispersion showing the kink based on the same Eliashberg function.
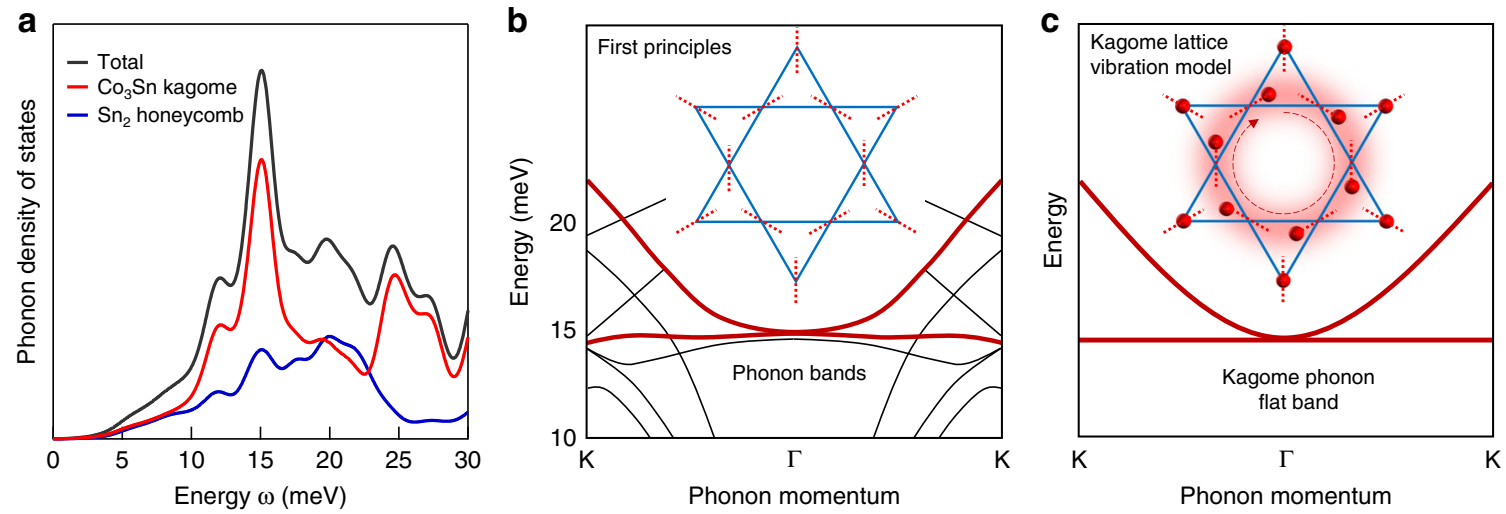

Fig. 4 Kagome flat-band origin of the bosonic mode. a Calculated layer-resolved phonon density of states, showing a pronounced peak at 15 meV arising from the $\mathrm{Co}_{3} \mathrm{Sn}$ kagome layer. $\mathbf{b}$ Calculated phonon band structure, showing a flat-band at $15 \mathrm{meV}$. The inset shows the calculated atomic displacements for the kagome lattice associated with the phonon flat-band, where the dotted lines denote the atomic vibration directions. $\mathbf{c}$ Phonon band structure for the kagome lattice vibration model. The inset shows the collective atomic vibrations associated with the phonon flat-band.

momentum space as shown in Fig. 4b. Lastly, through the atomic displacement resolved calculation, we identify that the flat-band phonon is mainly associated with the Co kagome lattice vibrations confined to the line connecting the centers of two neighboring triangles (Fig. 4b, inset).

In light of the first-principle calculation, we build a kagome lattice vibration model to elucidate the striking physics. The essential momentum features of the flat-band can be well captured by this model (Fig. 4c), with the flat-band toughing a parabolic band bottom. This model is highly analytical and offers a heuristic understanding of the non-propagating nature of the kagome flat-band phonon mode. We find that the collective lattice displacement shown in the inset of Fig. $4 c$, a deformation from a hexagonal ring (inner six atoms) rotating clockwise or anti-clockwise, would not exert any net force on the outer atoms. Hence, such geometrically frustrated vibrations can be localized forming the phonon flat-band. It is also clear now that this phonon flat-band is the lattice analog of kagome electron flatband $^{8,13}$, whose quadratic band touching feature distinguishes them from the isolated flat-bands in heavy-fermion systems ${ }^{30}$ and the Dirac cone touching flat-bands in Moire lattices ${ }^{31}$. The coupling to the kagome phonon flat-band is not predicted by existing 
research papers but is highly anticipated to explain the giant fermion-boson interaction observed here. Our findings suggest that the flat phonon dispersion can be probed by future momentum-resolved phonon-sensitive scattering experiments including inelastic X-ray scattering and neutron scattering.

The correspondence between the kagome lattice, tunneling conductance, magnetic field response, double kink feature, selfenergy analysis, first-principles, and lattice vibration model provides strong evidence and conceptual framework for the fermion-boson interaction in a geometrically frustrated topological quantum material. The nontrivial kagome band structures have been widely observed in both fermionic and bosonic systems ${ }^{15,32}$, but their many-body interactions were rarely experimentally observed previously. We expect the latter to be quite general in many topological quantum materials with flatbands. Such fermion-boson interactions can be the driving force for future discovery of incipient density waves and superconductivity in kagome lattice materials through pressure tuning or chemical engineering. Although our research addresses the coupling of the dispersive electrons and the flat-band phonon, it would be interesting to explore in the future the intriguing possibility of coupling of the kagome flat electron band and flat phonon band when they are tuned to the similar energies.

\section{Methods}

Sample preparation. High-quality single crystals of CoSn were synthesized by the Sn flux method. The starting elements of Co (99.99\%), Sn (99.99\%) were put into an alumina crucible, with a molar ratio of $\mathrm{Co:Sn}=3: 20$. The mixture was sealed in a quartz ampoule under a partial argon atmosphere and heated up to $1173 \mathrm{~K}$, then cooled down to $873 \mathrm{~K}$ with $2 \mathrm{~K} / \mathrm{h}$. The CoSn single crystals were separated from the Sn flux by using a centrifuge.

Scanning tunneling microscopy characterization. Single crystals with size up to $2 \mathrm{~mm} \times 2 \mathrm{~mm} \times 1 \mathrm{~mm}$ were cleaved mechanically in situ at $77 \mathrm{~K}$ in ultra-high vacuum conditions, and then immediately inserted into the STM head, already at $\mathrm{He} 4$ base temperature $(4.2 \mathrm{~K})$. The magnetic field was applied with zero-field cooling. Tunneling conductance spectra were obtained with an Ir/Pt tip using standard lock-in amplifier techniques with a root mean square oscillation voltage of $0.3 \mathrm{meV}$ and a lock-in frequency of $977 \mathrm{~Hz}$. Topographic images were taken with tunneling junction set up: $V=-100 \mathrm{mV}, I=2-0.05 \mathrm{nA}$. The conductance maps are taken with tunneling junction set up: $V=-50 \mathrm{mV}, I=0.5 \mathrm{nA}$.

Transmission electron microscopy characterization. Thin lamellae were prepared by focused ion beam cutting using a ThermoFisher Helios NanoLab G3 UC DualBeam. All samples for experiments were polished by $2 \mathrm{kV}$ Ga ion beam to minimize the surface damage caused by the high energy ion beam. Transmission electron microscopy imaging, atomic resolution high-angle annular dark-field scanning transmission electron microscopy imaging and atomic-level energy-dispersive X-ray spectroscopy mapping were performed on a double Cs corrected ThermoFisher Titan Cubed Themis 300 scanning/transmission electron microscope equipped with an extreme field emission gun (X-FEG) source operated at $300 \mathrm{kV}$ and super-X energy-dispersive spectrometry system.
Many-body theory for bosonic mode coupling. Due to the scattering by bosonic modes, the electrons do not have a definite energy but rather a finite lifetime and a broadened spectral function. In many-body theory, this phenomenon is characterized by the electron self-energy $\Sigma(\omega)$, which can be regarded as a correction to the free-electron Green function. The electron Green function is

$$
G(k, \omega)=\frac{1}{\omega-\epsilon_{k}^{0}-\Sigma(\omega)},
$$

where $\epsilon_{k}^{0}$ is the electron bare energy dispersion based on first-principle calculation. And the electron spectral function is

$$
A(k, \omega)=\frac{1}{\pi}|\operatorname{Im} G(k, \omega)| .
$$

The QPI dispersion is further described by

$$
Q(q, \omega)=-\frac{1}{\pi} \sum_{k} \operatorname{Im} G(q+k, \omega) \mathrm{G}(k, \omega) .
$$

With the Eliashberg function, the electron self-energy can be described by

$$
\Sigma(\omega)=\int \mathrm{d} E \int \mathrm{d} \omega^{\prime} \alpha^{2} F\left(\omega^{\prime}\right)\left[\frac{1-f(E)+n\left(\omega^{\prime}\right)}{\omega-\omega^{\prime}-E}+\frac{f(E)+n\left(\omega^{\prime}\right)}{\omega+\omega^{\prime}-E}\right],
$$

where $\alpha^{2} F(\omega)$ is Eliashberg coupling function describing the electron-bosonic mode interaction, $f(E)$ is the Fermi-Dirac distribution, and $n(\omega)$ is Bose-Einstein distribution at temperature $T$. Since in most cases, the bosonic mode energy is far less than the Fermi energy $\varepsilon_{\mathrm{F}}$, we can make the approximation that the initial and final energies of scattered electrons are close to Fermi energy. In this way, we obtain an Eliashberg coupling function solely determined by the bosonic energy distribution. An effective coupling constant can be defined as

$$
\lambda=2 \int \frac{\alpha^{2} F(\omega)}{\omega} \mathrm{d} \omega .
$$

In our experiment, we found a kink in the electron dispersion at $E_{\mathrm{M}}= \pm 15 \mathrm{meV}$, which implies a dominant bosonic mode with $\omega_{\mathrm{E}}=15 \mathrm{meV}$. In the calculation, we use the Einstein model and take $\alpha^{2} F(\omega)$ as a gaussian peak centered at $\omega_{\mathrm{E}}$. The calculated $\operatorname{Re} \Sigma(k, \omega)$ is given in Fig. 3e, with comparison to experimental data. With the electron Green function, we can simulate the QPI dispersion $Q(q, \omega)$ by Eq. (3) convoluted with the experimental resolution, which is given in Fig. $3 \mathrm{f}$.

First-principles calculation. We perform electronic structure calculations within the framework of the density functional theory using norm-conserving pseudopotentials ${ }^{33}$ as implemented in the Quantum Espresso simulation package ${ }^{34}$. The exchange-correlation effects are treated within the local density approximation with the Perdew-Zunger parametrization ${ }^{35}$. The electronic calculations used a plane-wave energy cutoff of $80 \mathrm{Ry}$ and a $12 \times 12 \times 12 \Gamma$-centered $k$-mesh to sample the Brillouin zone. Total energies were converged to $10^{-7} \mathrm{Ry}$ in combination with Methfessel-Paxton type broadening of $0.01 \mathrm{Ry}$. The phonon calculation is done by using a $2 \times 2 \times 2 q$-mesh grid centered at $\Gamma$ for the sampling of phonon momenta Starting with the experimental structure, we have fully optimized both the ionic positions and lattice parameters until the Hellmann-Feynman force on each atom is $<10^{-3} \mathrm{Ry} /$ a.u. $\left(10^{-4} \mathrm{Ry}\right)$ and zero-stress tensors are obtained. We find that the flat-band phonon is mainly associated with the vibrations of $\mathrm{Co}$ atoms in $\mathrm{Co}_{3} \mathrm{Sn}$ lattice confined to the line connecting the centers of two neighboring triangles as shown by the dotted line in Fig. 5a. Figure 5b shows the atom displacement resolved phonon band structure.

Kagome lattice vibration model. We consider a kagome lattice vibration model, assuming the motion of the atoms is highly anisotropic and confined to the dotted line in Fig. 5a. The analysis reproduces the kagome band structure with a flat band quadratically touching another band.
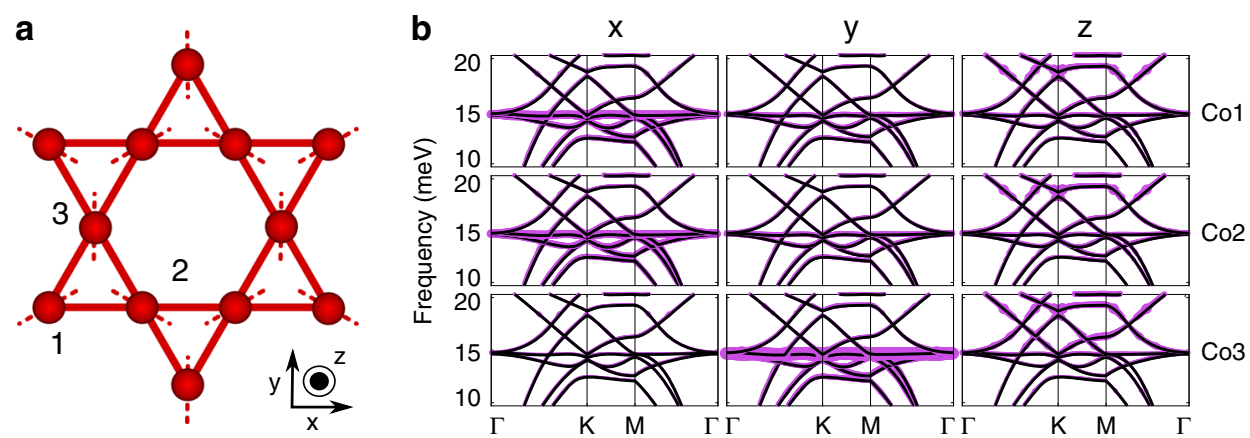

Fig. 5 Vibration nature of the phonon flat band. a Kagome lattice with dotted lines denoting the directions in which the atomic movement is confined. The inner atoms are displaced according to the elementary localized deformation that comprises the flat band. $\mathbf{b}$, Atom displacement resolved phonon band structure along $x, y$, and $z$-direction vibration for $\mathrm{CO}_{1}, \mathrm{CO}_{2}$, and $\mathrm{CO}_{3}$ corresponding to $\mathbf{a}$. The spectral weight is reflected by the line thickness. 
We choose the vectors in real space connecting nearest neighbor kagome atoms as

$$
\boldsymbol{a}_{1}=(1 / 2, \sqrt{3} / 2) \quad a_{2}=(1,0) \quad a_{3}=a_{1}-a_{2} .
$$

The three atoms $\alpha=1,2,3$ in each kagome unit cell can move along the directions

$$
\boldsymbol{U}_{1}=(\sqrt{3} / 2,1 / 2) \quad \boldsymbol{U}_{2}=(-\sqrt{3} / 2,1 / 2) \quad \boldsymbol{U}_{3}=(0,-1)
$$

All atoms are then coupled with the same spring constant $\beta$, except for the sign. The atoms in one unit cell have the potential energy

$$
E_{\text {intra }}=\frac{\beta}{2} \sum_{\boldsymbol{R}}\left[\left(\boldsymbol{U}_{1, \boldsymbol{R}}-\boldsymbol{U}_{2, \boldsymbol{R}}\right)^{2}+\left(\boldsymbol{U}_{1, \boldsymbol{R}}-\boldsymbol{U}_{3, \boldsymbol{R}}\right)^{2}+\left(\boldsymbol{U}_{2, \boldsymbol{R}}+\boldsymbol{U}_{3, \boldsymbol{R}}\right)^{2}\right]
$$

In contrast, the inter-unit cell coupling reads

$$
\begin{aligned}
E_{\text {inter }}= & \frac{\beta}{4} \sum_{\boldsymbol{R}}\left[\left(\boldsymbol{U}_{1, \boldsymbol{R}}-\boldsymbol{U}_{2, \boldsymbol{R}-2 \boldsymbol{a}_{2}}\right)^{2}+\left(\boldsymbol{U}_{1, \boldsymbol{R}}-\boldsymbol{U}_{3, \boldsymbol{R}-2 \boldsymbol{a}_{1}}\right)^{2}+\left(\boldsymbol{U}_{2, \boldsymbol{R}}+\boldsymbol{U}_{3, \boldsymbol{R}-2 \boldsymbol{a}_{3}}\right)^{2}\right. \\
& \left.+\left(\boldsymbol{U}_{2, \boldsymbol{R}}-\boldsymbol{U}_{1, \boldsymbol{R}+2 \boldsymbol{a}_{2}}\right)^{2}+\left(\boldsymbol{U}_{3, \boldsymbol{R}}-\boldsymbol{U}_{1, \boldsymbol{R}+2 \boldsymbol{a}_{1}}\right)^{2}+\left(\boldsymbol{U}_{3, \boldsymbol{R}}+\boldsymbol{U}_{2, \boldsymbol{R}+2 \boldsymbol{a}_{3}}\right)^{2}\right] .
\end{aligned}
$$

After Fourier transformation, the potential energy reads

$$
E_{\mathrm{pot}}=\sum_{k}\left(\boldsymbol{U}_{1, \boldsymbol{k}}, \boldsymbol{U}_{2, \boldsymbol{k}}, \boldsymbol{U}_{3, \boldsymbol{k}}\right) v_{k}\left(\begin{array}{c}
\boldsymbol{U}_{1,-\boldsymbol{k}} \\
\boldsymbol{U}_{2,-\boldsymbol{k}} \\
\boldsymbol{U}_{3,-\boldsymbol{k}}
\end{array}\right),
$$

with

$$
v_{k}=\frac{\beta}{2}\left(\begin{array}{ccc}
4 & -1-e^{2 i \boldsymbol{k} \cdot \boldsymbol{a}_{2}} & -1-e^{2 i \boldsymbol{k} \cdot \boldsymbol{a}_{1}} \\
-1-e^{-2 i \boldsymbol{k} \cdot \boldsymbol{a}_{2}} & 4 & 1+e^{2 i \boldsymbol{k} \cdot \boldsymbol{a}_{3}} \\
-1-e^{2 i \boldsymbol{k} \cdot \boldsymbol{a}_{1}} & 1+e^{-2 i \boldsymbol{k} \cdot \boldsymbol{a}_{3}} & 4
\end{array}\right) .
$$

The spectrum of $v_{k}$ is given by

$$
\omega_{k}^{2}=\frac{\beta}{m}\left\{1, \quad \frac{5}{2} \pm \frac{1}{2} \sqrt{3+2 \cos \left(\boldsymbol{k}_{1}\right)+2 \cos \left(\boldsymbol{k}_{1}-\boldsymbol{k}_{2}\right)+2 \cos \left(\boldsymbol{k}_{2}\right)}\right\},
$$

where $\boldsymbol{k}_{1}, \boldsymbol{k}_{2}$ stand for the inner product of $\boldsymbol{k}$ vector with $\boldsymbol{a}_{1}, \boldsymbol{a}_{2}$. We can observe the characteristic flat-band and two quadratic bands.

\section{Data availability}

All relevant data are available from the corresponding authors upon reasonable request.

Received: 23 March 2020; Accepted: 1 July 2020;

Published online: 10 August 2020

\section{References}

1. Keimer, B. \& Moore, J. E. The physics of quantum materials. Nat. Phys. 13, 1045-1055 (2017).

2. Broholm, C. et al. Quantum spin liquids. Science 367, eaay0668 (2020).

3. Xu, G., Lian, B. \& Zhang, S.-C. Intrinsic quantum anomalous Hall effect in the kagome lattice $\mathrm{Cs}_{2} \mathrm{LiMn}_{3} \mathrm{~F}_{12}$. Phys. Rev. Lett. 115, 186802 (2015)

4. Nakatsuji, S., Kiyohara, N. \& Higo, T. Large anomalous Hall effect in a non-collinear antiferromagnet at room temperature. Nature 527, 212-215 (2015).

5. Kuroda, K. et al. Evidence for magnetic Weyl fermions in a correlated metal. Nat. Mater. 16, 1090-1095 (2017).

6. Ye, L. et al. Massive Dirac fermions in a ferromagnetic kagome metal. Nature 555, 638-642 (2018).

7. Yin, J.-X. et al. Giant and anisotropic spin-orbit tunability in a strongly correlated kagome magnet. Nature 562, 91-95 (2018).

8. Yin, J.-X. et al. Negative flat band magnetism in a spin-orbit-coupled correlated kagome magnet. Nat. Phys. 15, 443-448 (2019).

9. Wang, Q. et al. Large intrinsic anomalous Hall effect in half-metallic ferromagnet $\mathrm{Co}_{3} \mathrm{Sn}_{2} \mathrm{~S}_{2}$ with magnetic Weyl fermions. Nat. Commun. 9, 3681 (2018).

10. Zhang, S. S. et al. Many-body resonance in a correlated topological kagome antiferromagnet. Preprint at https://arxiv.org/abs/2006.15770 (2020).

11. Yin, J. -X. et al. Discovery of a quantum limit Chern magnet $\mathrm{TbMn}_{6} \mathrm{Sn}_{6}$. Preprint at https://arxiv.org/abs/2006.04881 (2020).

12. Kang, M. et al. Dirac fermions and flat bands in the ideal kagome metal FeSn. Nat. Mater. 19, 163-169 (2020).

13. Liu, H. et al. Orbital-selective dirac fermions and extremely flat bands in the nonmagnetic kagome metal CoSn. Preprint at https://arxiv.org/abs/ 2001.11738 (2020).
14. Raghu, R. \& Haldane, F. D. M. Analogs of quantum-Hall-effect edge states in photonic crystals. Phys. Rev. A 78, 033834 (2008).

15. Ozawa, T. et al. Topological photonics. Rev. Mod. Phys. 91, 015006 (2019).

16. Larsson, M. et al. Specific heat measurements of the FeGe, FeSn and CoSn compounds between 0.5 and 9 K. Phys. Scr. 9, 51-52 (1974).

17. McMillan, W. L. \& Rowell, J. M. Lead phonon spectrum calculated from superconducting density of states. Phys. Rev. Lett. 14, 108 (1965).

18. Lee, J. et al. Interplay of electron-lattice interactions and superconductivity in $\mathrm{Bi}_{2} \mathrm{Sr}_{2} \mathrm{CaCu}_{2} \mathrm{O}_{8+\delta}$. Nature 442, 546-550 (2006).

19. Shan, L. et al. Evidence of a spin resonance mode in the iron-based superconductor $\mathrm{Ba}_{0.6} \mathrm{~K}_{0.4} \mathrm{Fe} 2 \mathrm{As}_{2}$ from scanning tunneling spectroscopy. Phys. Rev. Lett. 108, 227002 (2012).

20. Migdal, A. Interaction between electrons and lattice vibrations in a normal metal. Sov. Phys. JETP 7, 996 (1958).

21. Engelsberg, S. \& Schrieffer, J. R. Coupled electron-phonon system. Phys. Rev. 131, 993 (1963).

22. Hlobil, P. et al. Tracing the electronic pairing glue in unconventional superconductors via inelastic scanning tunneling spectroscopy. Phys. Rev. Lett. 118, 167001 (2017).

23. Lanzara, A. et al. Evidence for ubiquitous strong electron-phonon coupling in high-temperature superconductors. Nature 412, 510-514 (2001).

24. Bostwick, A. et al. Quasiparticle dynamics in graphene. Nat. Phys. 3, 36-40 (2007).

25. Grothe, S. Quantifying many-body effects by high-resolution Fourier Transform scanning tunneling spectroscopy. Phys. Rev. Lett. 111, 246804 (2013).

26. Wang, Z. et al. Quasiparticle interference and strong electron-mode coupling in the quasi-one-dimensional bands of $\mathrm{Sr}_{2} \mathrm{RuO}_{4}$. Nat. Phys. 13, 799-805 (2017).

27. Kondo, T. et al. Anomalous dressing of Dirac Fermions in the topological surface state of $\mathrm{Bi}_{2} \mathrm{Se}_{3}, \mathrm{Bi}_{2} \mathrm{Te}_{3}$, and Cu-Doped $\mathrm{Bi}_{2} \mathrm{Se}_{3}$. Phys. Rev. Lett. 110, 217601 (2013).

28. Shi, J. et al. Direct extraction of the Eliashberg function for electron-phonon coupling: a case study of Be(1010). Phys. Rev. Lett. 92, 186401 (2004).

29. Zhou, X. J. et al. Multiple bosonic mode coupling in the electron self-energy of $\left(\mathrm{La}_{2-\mathrm{x}} \mathrm{Sr}_{\mathrm{x}}\right) \mathrm{CuO}_{4}$. Phys. Rev. Lett. 95, 117001 (2005).

30. Si, Q. \& Steglich, F. Heavy Fermions and quantum phase transitions. Science 329, 1161-1166 (2010).

31. Bistritzer, R. \& MacDonald, A. H. Moiré bands in twisted double-layer graphene. Proc. Natl Acad. Sci. USA 108, 12233-12237 (2011).

32. Armitage, N. P., Mele, E. J. \& Vishwanath, A. Weyl and Dirac semimetals in three-dimensional solids. Rev. Mod. Phys. 90, 015001 (2018).

33. Troullier, N. \& Martins, J. L. Efficient pseudopotentials for plane-wave calculations. Phys. Rev. B 43, 1993 (1991).

34. Giannozzi, P. et al. Quantum espresso: a modular and open-source software project for quantum simulations of materials. J. Phys. 21, 395502 (2009).

35. Perdew, J. P. \& Zunger, A. Self-interaction correction to density-functional approximations for many-electron systems. Phys. Rev. B 23, 5048-5079 (1981).

\section{Acknowledgements}

We acknowledge insightful discussions with Biao Lian and Zhida Song, and technical assistance from Limin Liu and Gaihua Ye. Experimental and theoretical work at Princeton University was supported by the Gordon and Betty Moore Foundation (Grant No. GBMF4547 and GBMF9461/Hasan). Sample characterization was supported by the United States Department of Energy (US DOE) under the Basic Energy Sciences programme (Grant No. DOE/BES DE-FG-02-05ER46200). M.Z.H. acknowledges support from Lawrence Berkeley National Laboratory and the Miller Institute of Basic Research in Science at the University of California, Berkeley in the form of a Visiting Miller Professorship. This work benefited from partial lab infra-structure support under NSF-DMR1507585. M.Z.H. also acknowledges visiting scientist support from IQIMat the California Institute of Technology. The work at Renmin University was supported by the National Key R\&D Program of China (Grants Nos. 2016YFA0300504 and 2018YFE0202600), the National Natural Science Foundation of China (No. 11774423,11822412), the Fundamental Research Funds for the Central Universities, and the Research Funds of Renmin University of China (RUC) (18XNLG14, 19XNLG17). The authors acknowledge the use of Princeton's Imaging and Analysis Center, which is partially supported by the Princeton Center for Complex Materials, a National Science Foundation (NSF)-MRSEC program (DMR-1420541). T.-R.C. was supported from the Young Scholar Fellowship Program by the Ministry of Science and Technology (MOST) in Taiwan, under MOST Grant for the Columbus Program No. MOST109-2636-M-006-002, National Cheng Kung University, Taiwan, and National Center for Theoretical Sciences (NCTS), Taiwan. This work was supported partially by the MOST, Taiwan, grant MOST107-2627-E-006-001. This research was supported in part by Higher Education Sprout Project, Ministry of Education to the Headquarters of University. Z.W. and K.J. acknowledge US DOE Grant No. DEFG02-99ER45747. T.N. acknowledges support from the European Union's Horizon 2020 research and innovation programme (ERC-StG-Neupert-757867-PARATOP). The work performed at the Texas Center for Superconductivity at the University of Houston is 
supported by the U.S. Air Force Office of Scientific Research Grant FA9550-15-1-0236 the T.L.L. Temple Foundation, the John J. and Rebecca Moores Endowment, and the State of Texas through the Texas Center for Superconductivity at the University of Houston. R.H. acknowledges support by NSF CAREER Grant No. DMR-1760668 and NSF MRI Grant No. DMR-1337207.

\section{Author contributions}

J.-X.Y., N.S., S.S.Z., and Y.J. conducted tunneling experiments in consultation with M.Z.H; Q.W., D.W., and H.L. synthesized and characterized the sequence of samples; S.M., H.-J.T., G.Chang, K.J., Z.W., T.N., A.A., and T.-R.C. carried out theoretical analysis in consultation with J.-X.Y. and M.Z.H.; D.M., G.Cheng, N.Y., S.W., L.D., Z.Y., R.H., Z.L., and C.-W.C. contributed to sample characterization. J.-X.Y., N.S., and M.Z.H. performed the data analysis and figure development, and wrote the paper with contributions from all authors; M.Z.H. supervised the project. All authors discussed the results, interpretation, and conclusion.

\section{Competing interests}

The authors declare no competing interests.

\section{Additional information}

Correspondence and requests for materials should be addressed to J.-X.Y. or M.Z.H.
Peer review information Nature Communications thanks Jorge Facio, Zhenyu Zhang and the other, anonymous, reviewer(s) for their contribution to the peer review of this work.

Reprints and permission information is available at http://www.nature.com/reprints

Publisher's note Springer Nature remains neutral with regard to jurisdictional claims in published maps and institutional affiliations.

\section{(c) (i)}

Open Access This article is licensed under a Creative Common Attribution 4.0 International License, which permits use, sharing, adaptation, distribution and reproduction in any medium or format, as long as you give appropriate credit to the original author(s) and the source, provide a link to the Creative Commons license, and indicate if changes were made. The images or other third party material in this article are included in the article's Creative Commons license, unless indicated otherwise in a credit line to the material. If material is not included in the article's Creative Commons license and your intended use is not permitted by statutory regulation or exceeds the permitted use, you will need to obtain permission directly from the copyright holder. To view a copy of this license, visit http://creativecommons.org/ licenses/by/4.0/.

(c) The Author(s) 2020 